\title{
A MECHANISM FOR LINEAR INSTABILITY IN TWO-DIMENSIONAL RIMMING FLOW
}

\author{
BY
}

\author{
S. B. G. O'BRIEN
}

Department of Mathematics, University of Limerick, Ireland

\begin{abstract}
In rimming flow, a thin film of viscous liquid coats the inside of a cylinder whose axis is horizontal and which is rotating with constant angular velocity. It has been established experimentally that such flows are often unstable with a variety of secondary flow regimes having been observed experimentally [15]. We use a lubrication approximation extended to the first order in the dimensionless film thickness (including the small effects of the variation of the film pressure across its thickness and the surface tension) and study the stability of the steady solutions to two-dimensional disturbances. The modified evolution equation is found to have both asymptotically stable and unstable solutions arising from the pressure terms. Surface tension effects place a restriction on the critical wave number when instability occurs: in many cases, surface tension prevents instability.
\end{abstract}

1. The physical problem. The occurrence of instabilities in the flow of a thin film of viscous liquid placed in a cylindrical tube of radius $R$ whose axis is horizontal and which is rotating with constant angular velocity $\omega$ (Fig. 1) has attracted considerable interest in the scientific literature [1]-[4]. Such flows are sometimes referred to as rimming flows. The liquid is entrained on the cylinder wall and, given suitable wetting properties, a continuous film can be obtained. Previous authors have investigated this problem both experimentally and theoretically and some progress has been made. In many cases, it is found experimentally that the flow appears to be unstable to both two- and threedimensional disturbances [15]. One well-known example of instability arises when the liquid, instead of settling into a configuration that is uniform in the axial direction, settles into a number of wet and (relatively) dry areas (or rings) along the length of the cylinder. So striking are these features that the term "hygrocysts" [1] has been coined to describe this apparently stable secondary state. Visually, the effect vaguely resembles the well-known results obtained by G. I. Taylor for the flow of a viscous liquid in the annular region between two rotating cylinders (see, e.g., [3]).

Flows of this type obviously have industrial applications, a typical example being the process of coating the inside of cylindrical fluorescent light bulbs. Such a bulb consists of

Received May 22, 2000.

2000 Mathematics Subject Classification. Primary 76D07, 76E17, 76D08, 76B45.

Key words and phrases. Rimming flow, instability, lubrication theory, surface tension. 


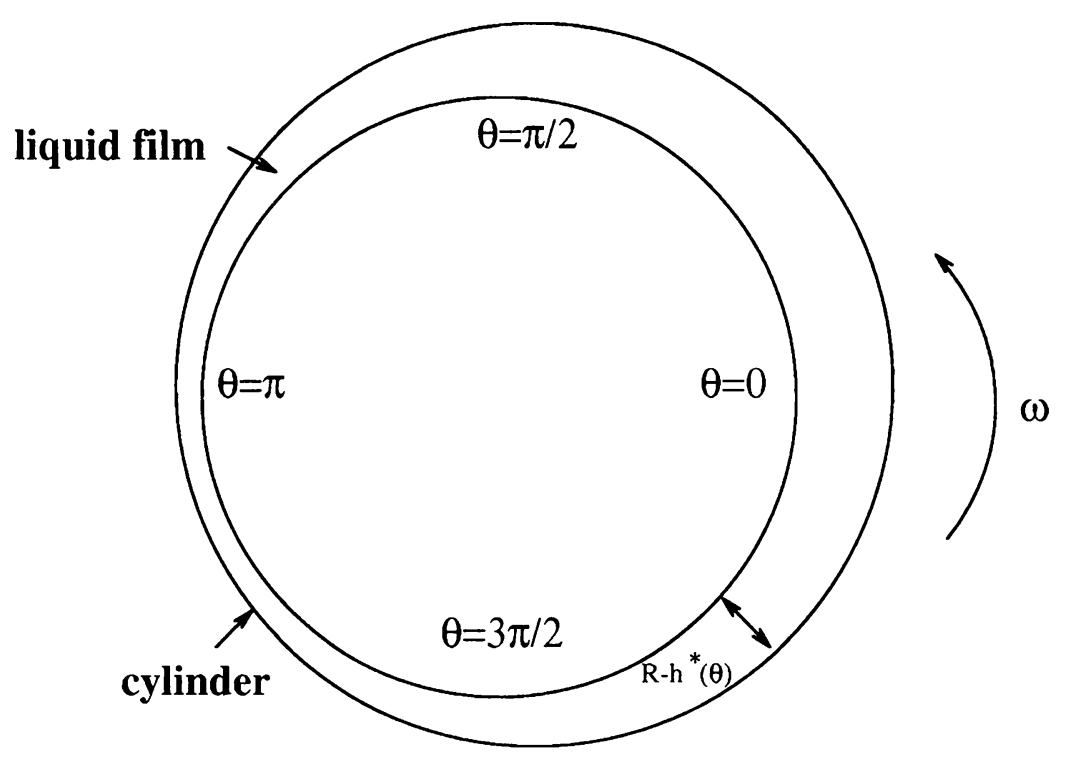

FIG. 1. Rotating cylinder in cross section

a hollow cylindrical glass tube coated with a thin layer of submicron phosphor particles, which give the light from the tube its characteristic colour. To obtain a uniform coating of this type, the particles are suspended in an inert liquid (e.g., water) and the resulting liquid suspension is used to coat the inside of the tube. If a suitably uniform suspension coating is attained, the excess liquid is encouraged to evaporate off (for example, by the strategic location of heating elements), the end result being a (uniform) coating of phosphor particles. From a practical point of view, if flow instabilities occur during the coating process, the resulting phosphor coating will be useless. A stability analysis is thus required in order to understand how instabilities might arise and, if possible, to deduce the critical values of the physical parameters at which the flow becomes unstable.

Despite the considerable theoretical attention [9], [4] that has been paid to this problem, no instability mechanisms have yet been pinpointed. In a recent paper [10], we took a useful step forward in realizing that the steady solutions could be written in closed form. The discovery of such closed-form solutions makes a stability analysis much more amenable from a mathematical point of view, and this omission by previous authors is apparently the reason why this sort of study has not previously been carried out. The other advantage of our approach to the problem lies in the non-dimensionalisation adopted, which we introduced previously [11] and which is more instructive than that used by previous authors, e.g., [9], [4]. Nevertheless, the work of Moffat [9] and Johnson [4] in identifying the main features of rimming flow must be acknowledged and, in particular, the work of Johnson in noticing the relevance of the discontinuous solutions. Johnson did not address the stability of these flows, which is the main point of this paper. For convenience we will follow here the development of [11], which classified the steady solutions according to the value of a single dimensionless parameter (the dimensionless film 
thickness). With this approach, when the dimensionless film thickness $H_{0}<0.7071$, the steady solutions are smooth and the flux $q<2 / 3$. If $H_{0} \geq 0.7071$, shocks form and the solution can be obtained from a single master curve [10]. In [16], the evolution equation governing the flow was solved numerically, including the effects of surface tension, and the smoothing effects of such terms near shocks was nicely illustrated.

In [12], we considered the linear stability of the steady solution resulting from a leading-order lubrication analysis. Attention was restricted to two-dimensional flows. In particular, steady solutions that were smooth were found to be only neutrally stable. Solutions with shocks in the first quadrant (which have been observed experimentally [15]) were also investigated, and it was suggested that such solutions should not give rise to flow instabilities. In order to further emphasize this point, we mathematically demonstrate the asymptotic stability of these physically relevant shock solutions (for all parameter values), concluding that the search for an instability mechanism must take a different direction. This is an obvious motivation to sharpen the approximation in search of "small" effects that might cause instability. In addition, it has been observed experimentally that instabilities often take a while to develop and occur for parameter values not consistent with shock solutions. Though somewhat imprecise, this suggests that the instabilities might arise as the result of small effects accumulating over a relatively long time period. To this end, we consider here a higher-order version of the lubrication approximation [13], [14] where the next order of "small" terms is included in a search for the instability mechanism. Since the leading-order shock solutions are asymptotically stable, we can restrict attention to smooth steady solutions. We should comment that having failed [12] to find a mechanism for instability at leading order, one might be inclined instead to immediately extend the search and include three-dimensional disturbances. Some of the instabilities observed (e.g., the aforementioned "rings") are clearly threedimensional effects. However, as the present paper suggests, it is the higher-order "small" terms that provide a mechanism for instability and it is sensible to restrict attention to two-dimensional disturbances in the first instance. We are also motivated to initially restrict attention to two-dimensional disturbances by the following factors. First, as we have already observed above, in many instances the leading-order approximation yields only a result of neutral stability, i.e., the solutions are not asymptotically stable. Second, we are motivated by consideration of Kuramoto-Sivashinsky type equations [7] discussed in $§ 5.3$. The introduction of higher-order terms in thin film flow [11] is known to introduce third- and fourth-order derivative terms and, as we shall see in the development, the second derivative terms can have a destabilizing effect. We will thus investigate the stability of this axially independent steady state film thickness to small two-dimensional disturbances using a first-order lubrication approximation to the equations of motion.

We will consider the linear stability (subject to two-dimensional disturbances) of the flow of a viscous liquid with kinematic viscosity $\nu$, (dynamic viscosity $\mu$ ), density $\rho_{l}$, in a long cylindrical tube of radius $R$, rotating at constant angular velocity $\omega$. The liquid volume fraction (assumed small) will be denoted by $V$ and the acceleration due to gravity by $g$. As a base steady state (see Fig. 1), we will use the closed-form steady solutions previously obtained [10] but extended to a higher order by using a regular perturbation procedure. 
2. The mathematical problem. We formulate the problem using polar coordinates $r^{*}$ and $\theta$ with origin on the cylinder's axis (see Fig. 1). It is more convenient to fix the coordinate axes rather than allow the axes to rotate with the cylinder. The dimensional film thickness is then given by $R-h^{*}\left(\theta, t^{*}\right)$. The relevant flow equations are the Stokes equations:

$$
0=-\nabla p^{*}+\mu \nabla^{2} \mathbf{u}^{*}+\rho_{1} \mathbf{g}
$$

where $p^{*}\left(r^{*}, \theta, t^{*}\right)$ is the pressure, $\mathbf{u}^{*}\left(r^{*}, \theta, t^{*}\right)$ is the liquid velocity vector, and $\mathbf{g}$ is the gravity vector. In addition, we assume the liquid is incompressible and hence the velocity vector must satisfy $\nabla \cdot \mathbf{u}^{*}=0$. We impose the following boundary conditions:

$$
\mathbf{u}^{*}=\left(u^{*}, v^{*}\right)=(0, \omega R) \text { on } r^{*}=R
$$

while on the liquid free surface, $r^{*}=h^{*}\left(\theta, t^{*}\right)$, we assume that the air exerts zero tangential and normal stress on the liquid while the surface tension gives rise to a jump in pressure across the interface, i.e.,

$$
\begin{aligned}
& \mathbf{n}^{*} \cdot \mathbf{T} \cdot \mathbf{n}^{*}=\gamma \kappa, \\
& \mathbf{t}^{*} \cdot \mathbf{T} \cdot \mathbf{n}^{*}=0,
\end{aligned}
$$

where $\mathbf{n}^{*}$ and $\mathbf{t}^{*}$ are unit normal and tangent vectors to the liquid free surface, $\mathbf{T}$ is the liquid stress tensor, and $\kappa$ is twice the mean curvature. We note that the free surface is defined parametrically by the vector-valued function $\mathbf{r}^{*}=h^{*}(\theta) \mathbf{e}_{r}$, where $\mathbf{e}_{r}$ is the unit vector in the radial direction. If $s$ is the arclength, then $\frac{d s}{d \theta}=\sqrt{h^{* 2}+h_{\theta}^{* 2}}$ and the curvature $\kappa$ is easily found to be

$$
\kappa=\frac{-\left(h^{* 2}+2 h_{\theta}^{* 2}\right)+h^{*} h_{\theta \theta}^{*}}{\left(h^{* 2}+h_{\theta}^{* 2}\right)^{3 / 2}} .
$$

In addition, we apply the kinematic condition on the unknown free surface, i.e.,

$$
\frac{D}{D t^{*}}\left(r^{*}-h^{*}\right)=0 \quad \text { on } r^{*}=h^{*}
$$

or, equivalently,

$$
h^{*} h_{t^{*}}^{*}=q_{\theta}^{*}
$$

where $q^{*}$ is the dimensional liquid flux

$$
\int_{r^{*}=h^{*}}^{r^{*}=R} v^{*} d r^{*}
$$

The average film thickness is given by

$$
H_{0}^{*}=\frac{1}{2 \pi} \int_{0}^{2 \pi}\left(R-h^{*}(\theta)\right) d \theta .
$$


2.1. The scaled problem. We non-dimensionalize as follows:

$$
u^{*}=\varepsilon U u, v^{*}=\omega R+U v, h^{*}=R-h H, r^{*}=R-\rho H, p^{*}=\frac{\mu U R}{H^{2}} p, t^{*}=t T,
$$

where

$$
U=\frac{\rho_{l} g H^{2}}{\mu}, \quad T=\frac{\mu R}{\rho_{l} g H^{2}},
$$

and $H$ is a typical film thickness, discussed below. In addition, we define $\varepsilon=H_{0}^{*} / R$ and we will assume that $H_{0}^{*} \ll R$ while noting that the volume fraction

$$
V=\frac{2 R H_{0}^{*}-H_{0}^{* 2}}{R^{2}}=2 \varepsilon-\varepsilon^{2}
$$

so the assumption of a small volume fraction $V \ll 1$ is approximately equivalent to the assumption that $\varepsilon \ll 1$. In real coating applications, like those referred to in the introduction, $V$ will typically be quite small; so we will use $\varepsilon$ as a small parameter in a perturbation expansion.

There are two possible choices for the characteristic film thickness $H$. The "natural" choice in a thin film approximation [11] is to use the average film thickness, i.e., to let $H \equiv H_{0}^{*}$. The alternative is discussed below. With this choice, the average dimensionless film thickness becomes $H_{0}=1$. We also define a related parameter

$$
H_{1}=\frac{H}{R} \delta^{-1}, \quad \delta^{2}=\frac{\omega \nu}{g R},
$$

where $\delta$ is a dimensionless rotation rate. For the asymptotic development that follows, we require $H_{1}$ to be an $O(1)$ dimensionless parameter.

The scaled problem to $O(\varepsilon)$ takes the form:

$$
\begin{aligned}
-\varepsilon \omega^{2} R / g & =p_{\rho}-\varepsilon \sin \theta \\
0 & =v_{\rho \rho}-H_{1}^{2} \cos \theta-\varepsilon v_{\rho}-H_{1}^{2} p_{\theta}, \\
0 & =-u_{\rho}(1+\varepsilon \rho)+\varepsilon u+v_{\theta}
\end{aligned}
$$

with boundary conditions:

$$
\begin{aligned}
(u, v) & =0 \quad \text { on } \rho=0, \\
p & =-\varepsilon \sigma\left(h+h_{\theta \theta}\right) \quad \text { on } \rho=h, \\
\frac{\partial q^{*}}{\partial \theta} & =\frac{\rho g H^{3}}{\mu}(-1+\varepsilon h) h_{t} \quad \text { on } \rho=h,
\end{aligned}
$$

where we have introduced a Bond number $\sigma=\gamma /\left(\rho_{l} g R^{2}\right)$. Following [12], and extending to the next order $u=u_{0}+\varepsilon u_{1}, v=v_{0}+\varepsilon v_{1}$, etc., the following solutions are obtained to $O(\varepsilon)$ (if we recall that $H_{1}$ is an $O(1)$ constant):

$$
p_{0}=0, \quad p_{1}=\varepsilon\left(\sin \theta-\omega^{2} R / g\right)(\rho-h)-\varepsilon \sigma\left(h+h_{\theta \theta}\right),
$$




$$
\begin{aligned}
& v_{0}=H_{1}^{2}\left(\cos \theta\left(\frac{\rho^{2}}{2}-h \rho\right)\right) \\
& v_{1}=H_{1}^{2}(-\rho+\left(\frac{1}{3} \rho^{3}+\frac{3}{2} h^{2} \rho-\rho^{2} h\right) \cos \theta \\
&\left.+\left(h \rho-\frac{1}{2} \rho^{2}\right)\left(h_{\theta}\left(\sin \theta-\frac{\omega^{2} R}{g}\right)+\sigma\left(h_{\theta}+h_{\theta \theta \theta}\right)\right)\right), \\
& u_{0}=H_{1}^{2}\left(-\sin \theta\left(\frac{\rho^{3}}{6}-\frac{h \rho^{2}}{2}\right)-\frac{\rho^{2}}{2} \cos \theta h_{\theta}\right) .
\end{aligned}
$$

An alternative choice for the non-dimensionalization is to let $H=\left(\frac{\omega R \nu}{g}\right)^{1 / 2}[10]$, in which case the dimensionless film thickness becomes

$$
H_{0}=\frac{1}{2 \pi} \int_{0}^{2 \pi} h(\theta) d \theta=\varepsilon \delta^{-1}
$$

and $H_{1}=1$. Equation (22) thus suggests that $\delta=O(\varepsilon)$. Furthermore, in this instance the effective small parameter is $\frac{\varepsilon}{H_{0}}=\delta$ and the asymptotic development is dependent on $\delta \ll 1$ as was already suggested after (11).

From this point on, we will use the second non-dimensionalization with $H \equiv\left(\frac{\omega R \nu}{g}\right)^{\frac{1}{2}}$ and so $T=1 / \omega$. The dimensionless liquid flux is now [4]:

$$
\begin{aligned}
q & =h+\int_{0}^{h} v d \rho \\
& =h-\frac{h^{3}}{3} \cos \theta+\delta\left(-\frac{1}{2} h^{2}+\frac{1}{3} h^{3} h_{\theta}(\sin \theta-G)+\frac{1}{2} h^{4} \cos \theta+\frac{1}{3} \sigma h^{3}\left(h_{\theta}+h_{\theta \theta \theta}\right)\right),
\end{aligned}
$$

where $G=\omega^{2} R / g$ (a Froude number) is a measure of the relative importance of gravity and centrifugal effects. The kinematic free surface condition is equivalent to

$$
(1-\delta h) h_{t}+q_{\theta}=0 .
$$

On putting $h=h_{0}+\delta h_{1},(24)$ becomes

$$
\begin{gathered}
h_{0 t}+\left(h_{0}-\frac{1}{3} h_{0}^{3} \cos \theta\right)_{\theta}+\delta\left[h_{1 \theta}+h_{1 t}+h_{0}^{2} h_{1} \sin \theta-2 \cos \theta h_{0} h_{0 \theta} h_{1}-\cos \theta h_{0}^{2} h_{1 \theta}\right. \\
-h_{0} h_{0 t}-h_{0} h_{0 \theta}+2 h_{0}^{3} h_{0 \theta} \cos \theta-\frac{1}{2} h_{0}^{4} \sin \theta+h_{0}^{2} h_{0 \theta}^{2}(\sin \theta-G)+\frac{1}{3} h_{0}^{3} h_{0 \theta \theta}(\sin \theta-G) \\
\left.+\frac{1}{3} h_{0}^{3} h_{0 \theta} \cos \theta\right]+\delta \sigma\left(\frac{1}{3} h_{0}^{3}\left(h_{0 \theta \theta}+h_{0 \theta \theta \theta}\right)+h_{0}^{2} h_{0 \theta}\left(h_{0 \theta}+h_{0 \theta \theta \theta}\right)\right)=0 .
\end{gathered}
$$

At this point we note, in particular, the occurrence of terms like $\frac{1}{3} h_{0}^{3} h_{0 \theta \theta}(\sin \theta-G+\sigma)$ in (25). In the top half of the cylinder, i.e., $\theta \in(0, \pi), \sin \theta>0$, and such a term can give rise to negative or backward diffusion effects, which are an obvious source of instability (cf. $\left.h_{t}=-h_{x x}\right)$. Negative diffusion has the effect of reinforcing any disturbances that may occur. In the absence of any opposing effects, this will certainly give rise to instability. However, (25) does have two opposing effects. First, the parameter $G\left(=\omega^{2} R / g\right)$ will reduce the negative diffusion effects (and would remove them entirely if $G \geq 1$ ). Second, there are surface tension induced diffusion effects (i.e., fourth derivative terms) that are 
stabilizing. In practice the surface tension effects are more important since $G$ tends to be very small.

In order to quantify this discussion, we first need to consider time-independent solutions of (25).

3. Time-independent solutions. Steady solutions to the leading-order version of (25) (i.e., putting $\delta \equiv 0$ in (25)) have been derived in [9], [4], and [10]. We will follow the latter development, where it is shown that the solutions are classified by the value of the dimensionless parameter $H_{0}$. If $H_{0}>H_{c} \approx 0.7071$, the liquid flux $q$ attains its maximum value of $\frac{2}{3}$ and shock-like solutions can occur in the quadrant $\left(-\frac{\pi}{2}, 0\right]$. If $H_{0}<H_{c}$, the film profile is smooth, without any jumps. In industrial applications, the film of liquid is usually as thin as possible [8] and typically $H_{0}<H_{c}$ and the base state is smooth. Nevertheless, we demonstrate the asymptotic stability of the physically relevant solutions with shocks at leading order, suggesting that the shocks are apparently not a mechanism for instability.

We define

$$
h_{a}=\left(-\frac{3 q}{2 \cos \theta}+\sqrt{\frac{9 q^{2}}{4 \cos ^{2} \theta}-\frac{1}{\cos ^{3} \theta}}\right)^{\frac{1}{3}}-\left(\frac{3 q}{2 \cos \theta}+\sqrt{\frac{9 q^{2}}{4 \cos ^{2} \theta}-\frac{1}{\cos ^{3} \theta}}\right)^{\frac{1}{3}}
$$

and

$$
h_{b}=\frac{2}{\sqrt{\cos \theta}} \cos \left(\frac{\phi}{3}\right), \quad h_{c}=\frac{2}{\sqrt{\cos \theta}} \cos \left(\frac{\phi}{3}-\frac{2 \pi}{3}\right),
$$

where $\phi \equiv \arccos \left(-\frac{3 q}{2} \sqrt{\cos \theta}\right)$. Note that when $\cos \theta<0$, we can reproduce $h_{a}$ from $h_{c}$ if we use the complex definition of the inverse cosine, $\arccos z=-i \ln \left(z+\sqrt{z^{2}-1}\right)$, choose the principal value of the square root and the principal value of the log. So for $\cos \theta<0$, we can rename $h_{a}$ as $h_{c}[10]$.

If $H_{0}<H_{c}$, then $q<2 / 3[10], h(\theta)$ is smooth, even and periodic and given by

$$
\begin{aligned}
h_{0}(\theta) & =q & & \text { if } \cos \theta=0 \\
& =h_{c} & & \text { if } \cos \theta \neq 0 .
\end{aligned}
$$

If $H_{0} \geq H_{c}$, then $q=\frac{2}{3}$ and shocks can occur. In this case, the most relevant shock solution starts on the branch $h_{c}$ at $\theta=-\pi$, jumps vertically to the branch $h_{b}$ at $\theta=\theta_{j}$, and then moves (smoothly) back onto the branch $h_{c}$ at $\theta=0$. The location of the shock is determined by demanding that $(22)$ be satisfied [10]. We thus define:

$$
\begin{aligned}
h_{\mathrm{sh}}(\theta) & =h_{c}, \quad \\
& =h_{b}, \quad \theta_{j} \leq \theta<0<\theta_{j}, \\
& =h_{c}, \quad 0 \leq \theta \leq \pi .
\end{aligned}
$$

In addition, when $q=2 / 3$, there is a special case solution with a corner at $\theta=0$ (corresponding to a shock of zero height) defined by

$$
h_{\text {cor }}=h_{0}
$$

as defined in (28) with $q=2 / 3$. 
Other shock solutions are theoretically possible but apparently do not occur in practice [6]. The most obvious is the reflection of (29) in the $h$-axis. In addition, there is a possible double shock solution with the structure:

$$
\begin{aligned}
h_{\mathrm{sh} 2}(\theta) & =h_{c}, \quad-\pi \leq \theta<\theta_{j}, \\
& =h_{b}, \quad \theta_{j} \leq \theta<\theta_{l}, \\
& =h_{c}, \quad \theta_{l} \leq \theta \leq \pi,
\end{aligned}
$$

where $-\pi / 2<\theta_{j}<0, \pi / 2>\theta_{l}>0$. We will show in the next section that solutions such as (31) are unstable at leading order and this is consistent with the fact that such solutions are not observed in practice [6].

In order to proceed to higher order, let us thus consider the higher-order version of (25). We wish to examine the stability of steady solutions to this equation. Since we are assuming that $\varepsilon \ll 1$ and $H_{0}=O(1)$, then $\delta \ll 1$ and it is clear that we can seek a steady solution to $O(\delta)$ by treating the problem as a regular perturbation. Thus we assume that the flux $q$ is a given parameter and in the expression for the flux, we set

$$
h_{s}=h_{0}+\delta h_{1}+O\left(\delta^{2}\right),
$$

where $h_{s}$ is a steady solution to $O(\delta)$ and $h_{0}$ is as given in (28). It easily follows that

$$
h_{1}=\frac{\frac{1}{2} h_{0}^{2}-\frac{1}{3}(\sin \theta-G) h_{0}^{3} h_{0}^{\prime}-\frac{1}{2} h_{0}^{4} \cos \theta-\frac{1}{3} \sigma h_{0}^{3}\left(h_{0}^{\prime}+h_{0}^{\prime \prime \prime}\right)}{1-h_{0}^{2} \cos \theta},
$$

where primes denote derivatives with respect to $\theta$. A similar result was given in [4]. We note that, since we assume that the lower-order solution $h_{0}$ is smooth with well-behaved derivatives, the perturbation is a regular one.

4. Asymptotic stability of shock solutions. In order to emphasize the need to proceed to higher order, we note that the neutral stability of the zero-order smooth solutions has previously been addressed [12]. Here we first demonstrate the asymptotic stability of the zero-order solutions in the case where $H_{0}>H_{c}$ and a physically relevant stationary shock occurs in the quadrant $(-\pi / 2,0][15]$. At leading order the relevant evolution equation is (25) with $\delta \equiv 0$ and as a base steady solution we use $h_{\mathrm{sh}}(\theta)$ as defined in (29), which is a solution with shock in the quadrant $(-\pi / 2,0]$. Away from the shock we set

$$
h=h_{\mathrm{sh}}(\theta)+\tau \operatorname{Re}\left[f(\theta) e^{s t}\right],
$$

where $\tau \ll 1$. We substitute into the leading-order version of $(25)$ and, omitting terms of $O\left(\tau^{2}\right)$ or smaller, we obtain the following ordinary differential equation for $f(\theta)$ :

$$
f_{\theta}\left(1-h_{\mathrm{sh}}^{2} \cos \theta\right)+f\left(s-2 h_{\mathrm{sh}} \frac{d h_{\mathrm{sh}}}{d \theta} \cos \theta+h_{\mathrm{sh}}^{2} \sin \theta\right)=0
$$

with solution

$$
f\left(1-h_{\mathrm{sh}}^{2} \cos \theta\right)=A \exp \left(-s \int^{\theta} \frac{1}{\left(1-h_{\mathrm{sh}}^{2} \cos \theta\right)} d \theta\right) .
$$


From $(29)$ we note that $h_{\mathrm{sh}}(0)=1$. However, near $\theta=0$ we find that $1-h_{\mathrm{sh}}^{2} \cos (\theta) \sim$ $-2 \theta h_{\mathrm{sh}}^{\prime}(0)$ and so

$$
f\left(-2 \theta h_{\mathrm{sh}}^{\prime}(0)\right) \sim A \exp \left(-s \int^{\theta} \frac{1}{-2 \theta h_{\mathrm{sh}}^{\prime}(0)} d \theta\right)
$$

and we conclude that

$$
f \sim \frac{A}{2 h_{\mathrm{sh}}^{\prime}(0)} \theta^{\left(\frac{s}{2 h_{\mathrm{sh}}^{\prime}(0)}-1\right)} .
$$

We require that $f$ be finite at $\theta=0$; so we must have

$$
\frac{s}{2 h_{\mathrm{sh}}^{\prime}(0)} \geq 1 \text {. }
$$

From (29), we find that $h_{\mathrm{sh}}^{\prime}(0)=-1 / \sqrt{6}$; so we require $s \leq-\sqrt{2} / \sqrt{3}$. There is a continuous spectrum of strictly negative eigenvalues and the solutions are asymptotically stable. Physically, the shock absorbs any disturbances in its vicinity. It is thus necessary to proceed to a higher order to search for an instability mechanism. The perturbed shock speed to $O(\tau)$ satisfies the relationship

$$
c(t)=\frac{q^{-}-q^{+}}{h^{-}-h^{+}}=A \tau \exp (s t)
$$

where $A$ is defined by $q^{-}, q^{+}, h^{-}, h^{+}$, the values of the flux, and the film thickness on either side of the shock. We note in passing that solutions with a single shock in the quadrant $[0, \pi / 2]$ are unstable since in that case $h_{\mathrm{sh}}^{\prime}(0)=1 / \sqrt{6}$ and $s>0$.

The solution with a corner at the origin (30) is a special case. The analysis is similar to that given above in that the corresponding version of (36) still holds with $h_{\text {sh }}$ replaced by $h_{\text {cor }}$. In this instance, near $\theta=0$ it is clear that $h_{\text {cor }}^{\prime}(0)$ is not defined. However,

$$
h_{\text {cor }} \sim 1-\frac{|\theta|}{\sqrt{6}}, \text { i.e., } 1-h_{\text {cor }}^{2} \cos \theta \sim \frac{\sqrt{2}}{\sqrt{3}}|\theta|
$$

and we find that

$$
f \sim \frac{\sqrt{3}}{\sqrt{2}}|\theta|^{-s \frac{\sqrt{3}}{\sqrt{2}}-1}
$$

and we conclude that

$$
s \leq-\frac{\sqrt{2}}{\sqrt{3}}
$$

with consequent asymptotic stability.

For completeness, we briefly show that the other shock solutions are unstable at leading order and apparently do not occur in nature. The analysis of the stability of (31) is similar since (36) still holds with $h_{\mathrm{sh}}$ replaced by $h_{\mathrm{sh} 2}$. In this case $h_{\mathrm{sh} 2}$ has a corner at $\theta=0$ as in (31). However, near $\theta=0$,

$$
h_{\mathrm{sh} 2} \sim 1+\frac{|\theta|}{\sqrt{6}}, \text { i.e., } 1-h_{\mathrm{sh} 2}^{2} \cos \theta \sim \frac{-\sqrt{2}|\theta|}{\sqrt{3}}
$$


whence we deduce that

$$
f \sim \frac{-\sqrt{3}|\theta|^{s \frac{\sqrt{3}}{\sqrt{2}}-1}}{\sqrt{2}}
$$

and we conclude that

$$
s \geq \frac{\sqrt{2}}{\sqrt{3}}
$$

and the double shock solution is unstable at leading order.

Theoretical shock solutions are mathematically possible in the case where $q<2 / 3$ or $H_{0}<H_{c}$ as suggested by [4]. In this case only multiple shocks can occur. For example, suppose a double shock occurs as in (31) when $q<2 / 3$ with $\theta_{j}<\theta_{l} \in(-\pi / 2, \pi / 2)$. Then $h_{b}$ and $h_{c}$ do not touch at $\theta=0, h_{c}<1$ everywhere and, in addition, there is no corner at $\theta=0$; so an even number of shocks is required in $(-\pi / 2, \pi / 2)$. On the basis of kinematic wave theory, the kinematic wave speed (at leading order, see (25) with $\delta=0$ ) is given by $\frac{\partial q}{\partial h}=1-h^{2} \cos \theta$. If the shocks are located at $\theta_{j}$ and $\theta_{l}$, then $h=h_{b}$ in $\left[\theta_{j}, \theta_{l}\right)$. Now consider

$$
1-h_{b}^{2} \cos \theta=0 \Rightarrow \theta=\arccos \left(\frac{4}{9 q^{2}}\right)
$$

so there are no solutions for $q<2 / 3$. Thus the kinematic wave speed is

$$
\begin{array}{ll}
1-h_{c}^{2} \cos \theta>0, & \theta \in\left[-\pi, \theta_{j}\right), \\
1-h_{b}^{2} \cos \theta<0, & \theta \in\left[\theta_{j}, \theta_{l}\right), \\
1-h_{c}^{2} \cos \theta>0, & \theta \in\left[\theta_{l}, \pi\right] .
\end{array}
$$

Consider the shock at $\theta_{j}$. The kinematic wave speed on each side of the shock is towards the shock and we would expect disturbances to be swept up into it. However, the shock at $\theta_{l}$ has a kinematic wave speed that is away from the shock on both sides and small disturbances would tend to break up this shock with subsequent instability.

5. Linear stability analysis of smooth base solutions. Since none of the physically relevant solutions with shocks is neutrally stable, it is reasonable to restrict the investigation of the higher-order solutions to the smooth case, i.e., to test the stability of $h_{s}(\theta)$ as given in (32) as a steady solution of (25). We thus set

$$
h=h_{s}(\theta)+\tau f(\theta) e^{s t}
$$

where $\tau \ll 1$ and $f(\theta)$ is periodic with period $2 \pi$. Omitting terms of $O\left(\tau^{2}\right)$ or smaller, we obtain the following equation for $f(\theta)$ :

$$
a_{4}(\theta) f^{(i v)}+a_{3}(\theta) f^{\prime \prime \prime}+a_{2}(\theta) f^{\prime \prime}+a_{1}(\theta) f^{\prime}+a_{0}(\theta) f+s f=0,
$$


where $s$ are the (unknown) eigenvalues and the $a_{i}(\theta)$ are known functions with period $2 \pi$ defined as

$$
\begin{aligned}
a_{0}= & h_{0}^{2} \sin \theta-2 h_{0} h_{0}^{\prime} \cos \theta+\delta\left[2 h_{0} h_{1} \sin \theta-2 h_{0}^{\prime} h_{1} \cos \theta-2 h_{0} h_{1}^{\prime} \cos \theta-h_{0}^{\prime}\right. \\
& \left.+5 h_{0}^{\prime} h_{0}^{2} \cos \theta-h_{0}^{3} \sin \theta+2 h_{0} h_{0}^{\prime 2}(\sin \theta-G)+h_{0}^{2} h_{0}^{\prime \prime}(\sin \theta-G)\right] \\
& +\delta \sigma\left(2 h_{0} h_{0}^{\prime} h_{0}^{\prime \prime \prime}+h_{0}^{2} h_{0}^{\prime \prime \prime \prime}+2 h_{0} h_{0}^{\prime 2}+h_{0}^{2} h_{0}^{\prime \prime}\right), \\
a_{1}= & 1-h_{0}^{2} \cos \theta+\delta\left[-2 h_{0} h_{1} \cos \theta+\frac{4}{3} h_{0}^{3} \cos \theta+2 h_{0}^{2} h_{0}^{\prime}(\sin \theta-G)\right] \\
& +\delta \sigma h_{0}^{2}\left(h_{0}^{\prime \prime \prime}+2 h_{0}^{\prime}\right), \\
a_{2}= & \delta \frac{1}{3} h_{0}^{3}(\sin \theta-G+\sigma), \\
a_{3}= & \delta \sigma h_{0}^{2} h_{0}^{\prime} \\
a_{4}= & \delta \sigma \frac{1}{3} h_{0}^{3} .
\end{aligned}
$$

To attain the above expressions, we set $h_{s}=h_{0}+\delta h_{1}$ and expand while omitting terms of $O\left(\delta^{2}\right)$ or smaller. To test for instability, we need to see if any solutions of (49) exist for $f(\theta)$ with $\operatorname{Re}(s)>0$.

5.1. The numerical technique. We consider (49) where the known coefficients $a_{k}(\theta)$ are of period $2 \pi$ (as is $\left.h_{s}(\theta)\right)$. We write $a_{k}(\theta)$ and $f(\theta)$ as Fourier series:

$$
a_{k}(\theta)=\sum_{j=-\infty}^{\infty} a_{k}^{j} e^{i j \theta} ; \quad f(\theta)=\sum_{j=-\infty}^{\infty} C_{j} e^{i j \theta},
$$

where the $a_{k}^{j}$ are known constants given by

$$
a_{k}^{j}=\frac{1}{2 \pi} \int_{0}^{2 \pi} a_{k}(\theta) e^{-i j \theta} d \theta
$$

with $a_{k}^{-j}=\left(a_{k}^{j}\right)^{*}$. The constants $C_{j}$ in (55) are unknown as are the eigenvalues $s$ in (49). Noting that

$$
\sum_{j=-\infty}^{\infty} \alpha_{j} e^{i j \theta} \sum_{l=-\infty}^{\infty} \beta_{l} e^{i l \theta}=\sum_{m=-\infty}^{\infty} e^{i m \theta} \sum_{n=-\infty}^{\infty} \alpha_{m-n} \beta_{n}
$$

(49) becomes

$$
\sum_{k=0}^{k=4} \sum_{m=-\infty}^{m=\infty} e^{i m \theta} \sum_{n=-\infty}^{\infty} a_{k}^{m-n}(i n)^{k} C_{n}=-s \sum_{m=-\infty}^{m=\infty} C_{m} e^{i m \theta}
$$

and so it follows that

$$
\sum_{k=0}^{k=4} \sum_{n=-\infty}^{\infty} a_{k}^{m-n}(i n)^{k} C_{n}=-s C_{m}
$$

for all values $-\infty<m<\infty$. On defining

$$
\mathbf{f}=\left(\cdots C_{-1}, C_{0}, C_{1} \cdots\right)^{\mathrm{T}}
$$


and

$$
M_{m n}=\sum_{k=0}^{k=4} a_{k}^{m-n}(i n)^{k} \quad(-\infty<m, n<\infty),
$$

we can write (59) in the form

$$
\sum_{n=-\infty}^{\infty} M_{m n} C_{n}=-s C_{m}, \text { i.e., } \mathbf{M f}=-s \mathbf{f},
$$

where $\mathbf{M}$ is a square matrix of infinite order. Equation (62) is a matrix eigenvalue problem. In practice we truncate at some arbitrary integer value $N$ so that $-N \leq$ $m, n \leq N$ and (62) is approximated as a $(2 N+1) \times(2 N+1)$ matrix eigenvalue problem.

The numerical algorithm was successfully tested by considering the stability of (63), which is a type of Kuramoto-Sivashinsky equation [7] for which exact answers are available.

5.2. Numerical results. The most obvious conclusion from numerical investigations is that the current theory (unlike zero-order lubrication theory [12]) contains a mechanism for instability via negative diffusion. We note that there are four dimensionless parameters: $\delta, G, \sigma, H_{0}$, the first three of which correspond roughly to negative diffusion effects, centrifugal/gravity effects, and surface tension (Bond number) while $H_{0}$ parametrizes the steady solution being examined. Computations seem to indicate that if $\delta>0$ and $\sigma=G=0$, then solutions are always unstable since there are insufficient smoothing effects to neutralize the negative diffusion in (25). However, setting $\sigma=0$ makes the problem singularly perturbed in the sense that the highest derivative terms disappear.

Since diffusion effects are only destabilizing on a part of the domain, one might speculate on the existence of solutions with $\sigma=0$ that are stabilized by virtue of their being convected from the "dangerous" region of negative diffusion around to an interval where ordinary diffusion can stabilize (where $\sin \theta-G<0$ ). However, no such solutions were identified although our numerical search was certainly not exhaustive, but we suggest that such solutions cannot occur for the following reason. If negative diffusion occurs on any part of the domain, let us suppose that a disturbance occurs on that part of the domain with a very small wavelength. No matter how small the amplitude may be, in the absence of any smoothing, such a disturbance will always grow catastrophically before it is convected into the part of the domain where smoothing effects can occur if its wavelength is small enough.

If $G \geq 1+\sigma$, then $\sin \theta-G+\sigma \leq 0$, no negative diffusion can occur (see the term $\frac{1}{3} h_{0}^{3} h_{0 \theta \theta}(\sin \theta-G+\sigma)$ in $\left.(25)\right)$ and all solutions examined are found to be stable. More generally, if $\delta>0$, surface tension seems to have an overall stabilizing effect as illustrated in Fig. 2, at least in the realistic part of parameter space shown in the figure. Note that the surface tension contribution consists of two parts: a fourth-derivative term that is stabilizing (and that tends to make the free surface conformal with the underlying cylinder) and a second-derivative term that is destabilizing (and tends to make the free surface flat). In hte parameter region shown in Fig. 2, the marginal stability curves illustrate how surface tension effects can help neutralize the instability. Computations show that increasing $G$ also has a stabilizing effect. In practice, $G$ is small, and for this 


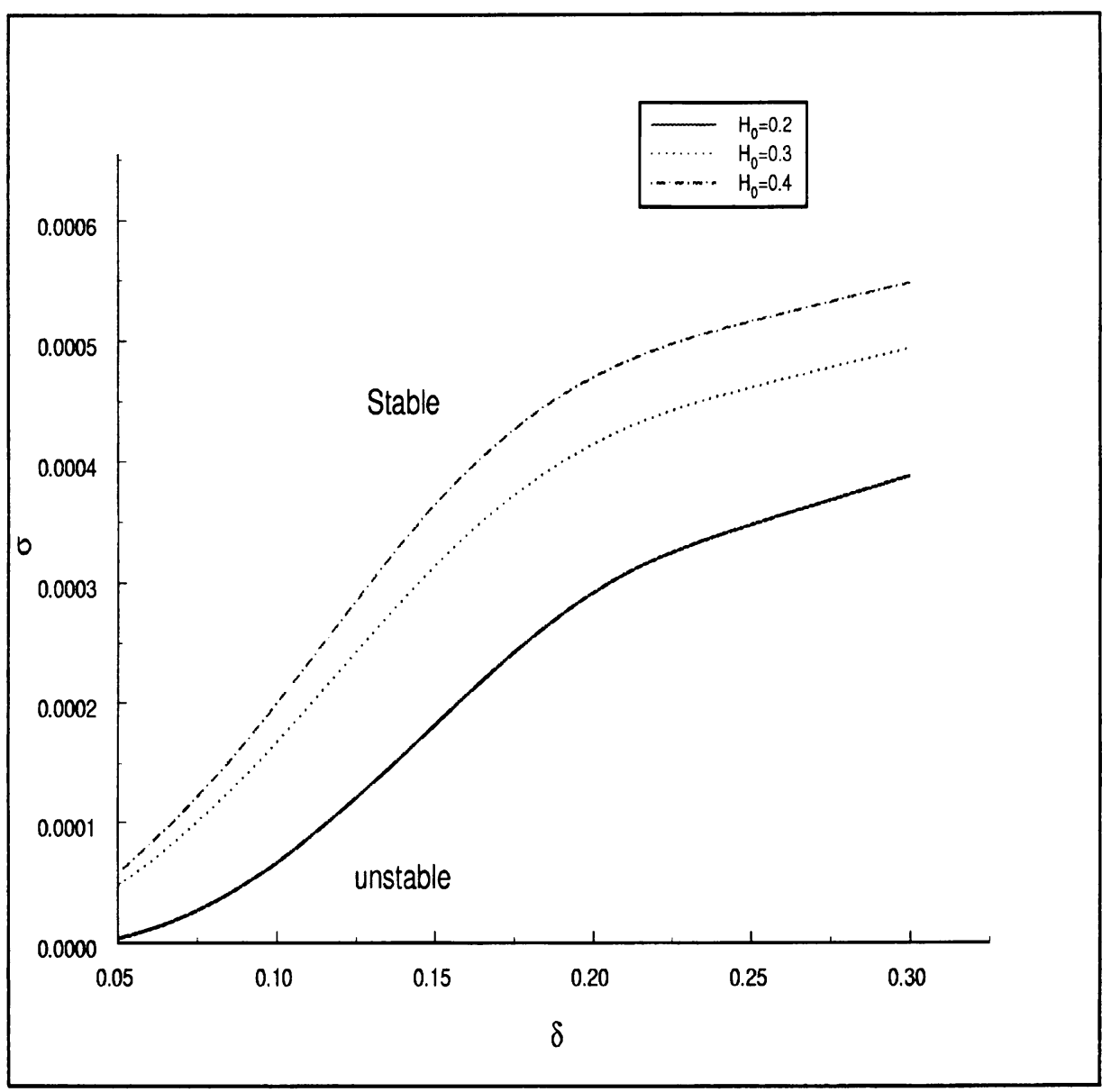

FIG. 2. Marginal stability curves for the cases $H_{0}=0.2,0.3,0.4, G=0$

reason most of the computations recorded here are for the case $G=0$. In Fig. 3 , we show how nonzero $G$ lowers the stability threshold for fixed $H_{0}$. In Fig. 2, the marginal stability curve passes through the origin, which follows from the fact that zero-order lubrication theory $(\delta, \sigma \equiv 0)$ yields solutions that are always neutrally stable [12]. While performing the computations necessary to draw the marginal stability curve in Fig. 2, we examined the associated eigenfunction when the eigenvalues were small in absolute value, making it difficult to decide whether the situation was stable or unstable. In clearly unstable situations, the eigenfunctions in all cases examined were found to take a form resembling that shown in Fig. 4, and this was used to make the decision in the borderline cases.

The effect of varying $H_{0}$ is illustrated in Fig. 2. We did not find any overall new trends in comparison to the results given here.

5.3. Comparison with the Kuramoto-Sivashinsky equation. It is worthwhile to compare the results in the present paper with a stability analysis of a Kuramoto-Sivashinsky 


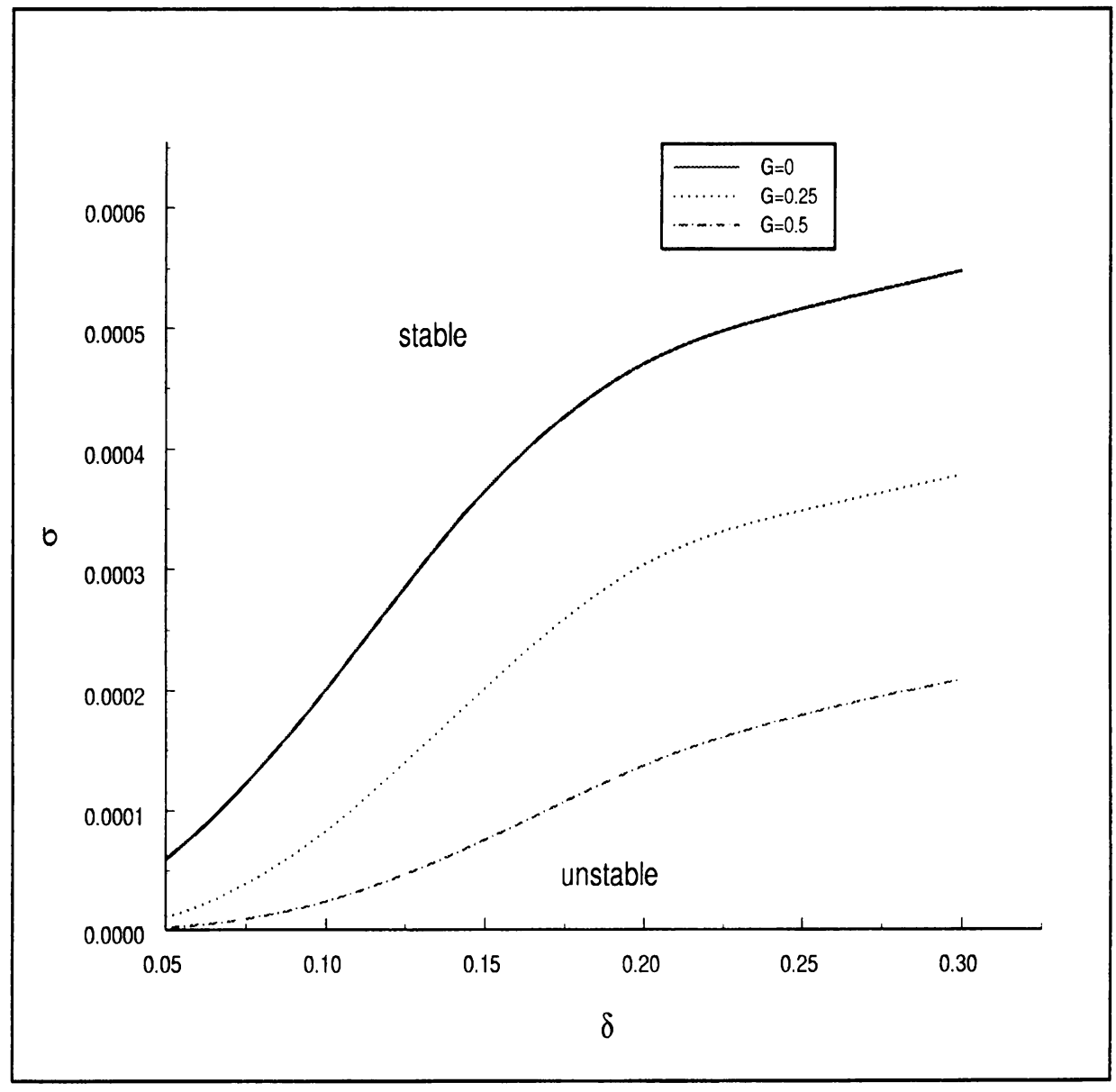

FIc. 3. Marginal stability curves for the cases $H_{0}=0.4, G=0,0.25,0.5$

type equation. For example, consider

$$
u_{t}+\beta u_{x x x x}+\alpha u_{x x}=0,
$$

where $\alpha, \beta$ are positive constants. On a finite domain with $x \in[0, l]$, the solution $u=$ 0 has a stability threshold based on a competition between the destabilizing negative diffusion effects of the second-derivative term and the stabilizing effects of the fourth derivative. The stability of $u=0$ can be tested by putting $u=\exp (s t) \exp (i k x)$, and we find that $u=0$ is a stable solution provided that $l<2 \pi \sqrt{\beta / \alpha}$, i.e., provided the domain is "short enough". If we consider a periodic version of $(63)$ on a fixed domain $[0,2 \pi], u=0$ is stable provided $1<\sqrt{\beta / \alpha}$. Generally speaking, the most dangerous disturbances are those with smaller wavenumbers. In terms of the wavenumber $k$, the unstable modes are in the interval $k<\sqrt{\alpha / \beta}$, i.e., the flow is unstable if $\sqrt{\alpha / \beta}>1$. In particular, if $\beta \ll \alpha$, there will be a range of unstable wavenumbers. The fastest-growing mode occurs when $s=\alpha k^{2}-\beta k^{4}$ attains a maximum, i.e., when $k=\frac{1}{\sqrt{2}} \sqrt{\frac{\alpha}{\beta}}$, which can 


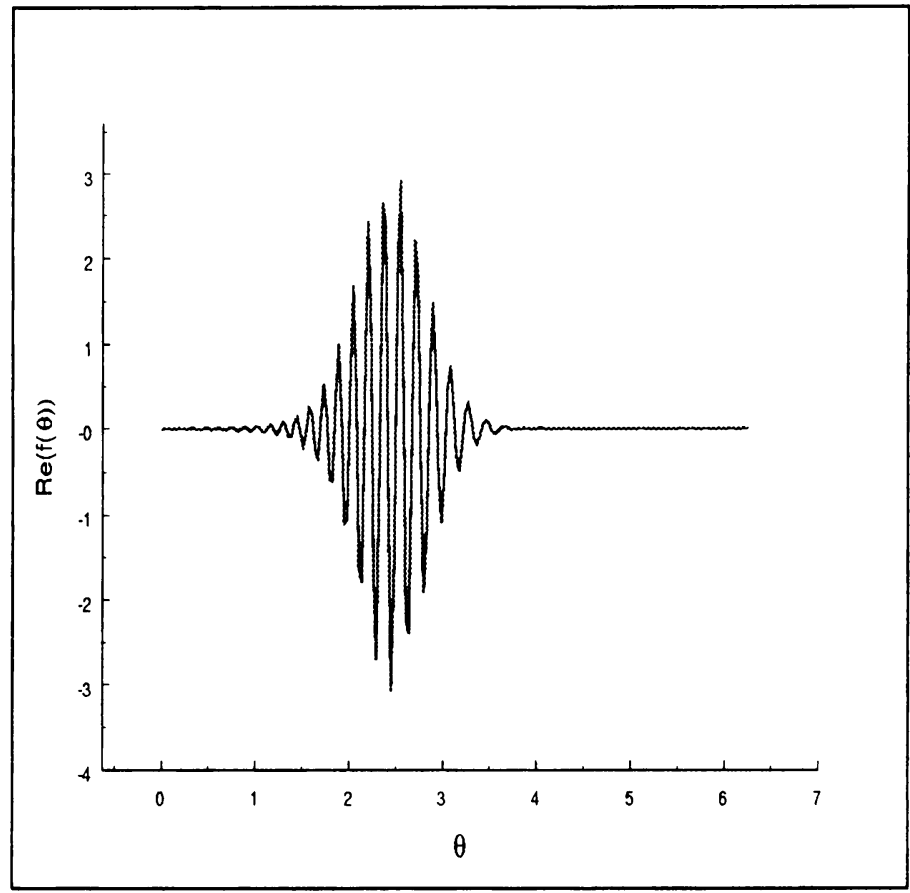

FIG. 4. The real part of the fastest-growing eigenfunction for the case $H=0.4, G=0, \sigma=0.0004, \delta=0.31$

be large if $\beta \ll \alpha$. If $\alpha \sim \beta$ and instability occurs, the unstable modes will all have $O(1)$ wavenumbers.

There are apparent similarities between (63) and (25), and a stability analysis of both equations displays similar trends. For example, the amplitude spectrum of an unstable mode for a typical realistic situation with $\sigma \ll 1$ is quite similar to that discussed above in the framework of (63) with $\beta \ll \alpha$. The fastest-growing mode has a considerable high frequency (wavenumber) content because the stabilizing terms are so small. For stable solutions we find that $O(1)$ wavenumbers predominate in the corresponding amplitude spectrum. In Fig. 4 we show computations for the eigenfunction corresponding to the fastest-growing mode for the case where $\sigma \ll 1$ and the oscillatory nature of this eigenfunction is an obvious result of the high-frequency components. We note also that oscillations originate in the region $[0, \pi]$ and are damped in $[\pi, 0]$ as we might expect: instability originates in the top of the cylinder and is diffused away in the lower half.

6. Flow on the outside of a rotating cylinder. It is worth noting that the development in the current paper is very similar to that required for a consideration of the flow on the outside of a rotating cylinder, which is the problem originally considered by Moffatt [9]. At lowest order the two problems are identical. At the next order, the most important difference will arise in the pressure gradient term. In the external problem, gravity will be stabilizing in $[0, \pi]$ and destablizing in $[-\pi, 0]$. Spin effects will be destabilizing everywhere rather than stabilizing everywhere in the internal (rimming) 
problem. Surface tension can play a stabilizing role, and presumably both stable and unstable cases will arise. Although the numerical results will be quantitatively different, we might expect the trends to be similar to those given here.

7. Conclusions and discussion. Using a leading-order lubrication theory, we previously showed [12] that the smooth steady solutions that occur when the dimensionless flux $q<\frac{2}{3}\left(H_{0}<0.7071\right)$ are neutrally stable. As pointed out in the introduction, it has been established experimentally that films of this type can become unstable. Since neutrally stable solutions are on the borderline between instability and asymptotic stability, an obvious progression was to try a higher-order lubrication approximation to see if the extra terms could push the steady solutions off the marginal stability curve, thus becoming either stable or unstable. This proved to indeed be the case: the higher-order lubrication approximation yields solutions of both types: (asymptotically) stable and unstable.

Generally speaking, the higher-order lubrication approximation gives rise to unstable solutions in the absence of other smoothing effects $(G=\sigma=0)$. This is physically easy to understand if we refer to (18): the higher-order lubrication approximation introduces the possibility of variations in pressure across the film thickness (leading-order lubrication theory assumes a constant pressure across the film thickness). In the top half of the cylinder where $\theta \in(0, \pi)$ (see Fig. 1), there is thus an obvious mechanism for instability and, in fact, this mechanism is similar to the mechanism by which droplets form in a thin film clinging to the underside of a horizontal plate. Small local increases in the film thickness give rise to a pressure gradient, which forces more liquid towards the disturbance and so it continues growing. The present problem is, however, more complicated in that there are also dynamic (convective) effects.

Mathematically, the destabilizing effects are traceable to the term $\sin \theta-G+\sigma$ in the evolution equation (25). If $G=0$, negative or backward diffusion effects (via the $\sin \theta$ term, typically of size $\delta$ ) always arise in the top half of the cylinder (although these effects are always stabilizing in the lower half) and can cause instability in the absence of any opposing stabilizing effects. If the parameter $G \geq 1+\sigma$, no negative diffusion can occur and the numerical results suggest that no instability arises. In the case of rimming flow, there are two stabilizing influences as measured by the parameters $G=\omega^{2} R / g$ and the Bond number $\sigma=\gamma /\left(\rho_{l} g R^{2}\right)$. Increasing $G$ is clearly stabilizing and this is physically easy to understand. This can be achieved by increasing the spin rate, which has the effect of raising the local centrifugal effects (which oppose the destabilizing second-order gravity effects in the liquid pressure). Surface tension effects are more complicated since they give rise to stabilizing fourth-derivative terms and destabilizing second-derivative terms, but in the physically relevant parameter regime, the stabilizing effects appear to dominate. Note that, since the flow is occurring on a finite domain $(0 \leq \theta \leq 2 \pi)$, not all wave numbers can occur as distinct from the case of a thin film clinging to the underside of an infinite horizontal plate, for example, where instability will always occur.

The model developed here is predictive and can be used to deduce whether or not a particular situation (with corresponding dimensionless parameters $\delta, H_{0}, G, \sigma$ ) is stable or not at least in-so-far as the lubrication theory approximates the flow. A nonlinear 
stability analysis would now be considerably simplified by the fact that the regions in parameter space that correspond to unstable solutions can be identified using the present linear stability theory. The present theory cannot be expected to predict the "hygrocysts" alluded to in the introduction since this is clearly a three-dimensional instability with axial $(z)$ variation. However, it is not impossible that the flow has a lower stability threshold to two-dimensional disturbances and this certainly makes the present study worthwhile. The three-dimensional analogue with axial variation thus require analysis, but at this point it is already clear that, on adding in $z$-dependent effects, there will at the very least exist a similar mechanism to that described in this paper which can lead to three-dimensional disturbances, i.e., there will be an analogous (small) instability mechanism arising as a result of pressure gradients, which will be liable to surface tension stabilizing effects.

\section{REFERENCES}

[1] R. T. Balmer and T. G. Wang, An experimental study of internal hygrocysts, Journal of Fluids Engineering, 688-693 (December 1976)

[2] R. T. Balmer, The hygrocyst, a stability phenomenon in continuum mechanics, Nature 227, 600-601 (1970)

[3] S. Chandrasekhar, Hydrodynamic and hydromagnetic stability, The International Series of Monographs on Physics, Clarendon Press, Oxford, 1961

[4] R. E. Johnson, Steady state coating flows inside a rotating horizontal cylinder, J. Fluid Mech. 190, 321-342 (1988)

[5] M. J. Karweit and S. Corrsin, Observation of cellular patterns in a partly filled, horizontal, rotating cylinder, Phys. Fluids 18, 111-112 (1975)

[6] J. P. Kovac and R. T. Balmer, Experimental studies of external hygrocysts, Journal of Fluids Engineering, 226-230 (June 1980)

[7] Y. Kuramoto, Diffusion induced chaos in reaction systems, Progress of Theoretical Physics 64, 346-367 (1978)

[8] J. Lammers, S. B. G. O'Brien, and M. N. M. Beerens, Proceedings of the first European symposium on The mechanics of thin film coatings, Leeds, 1995, pp. 397-403

[9] H. K. Moffatt, Behaviour of a viscous film on the outer surface of a rotating cylinder, Journal de Mécanique 16, 651-674 (1977)

[10] S. B. G. O'Brien and E. G. Gath, The location of a shock in rimming flow, Phys. Fluids 10, 1-3 (1998)

[11] S. B. G. O'Brien, Marangoni drying: Non-linear waves in a thin film, J. Fluid Mech. 254, 649-670 (1993)

[12] S. B. G. O'Brien, Linear stability of rimming flows, Quart. Appl. Math. LX, 201-211 (2002)

[13] L. W. Schwartz and D. E. Weidner, Modelling of coating flows on curved surfaces, J. Engrg. Math. 29, 91-103 (1995)

[14] J. A. Moriarty, L. W. Schwartz, and E. O. Tuck, Unsteady spreading of thin liquid films with small surface tension, Phys. Fluids A, 3, 733-742 (1993)

[15] J. L. Throne and J. Gianchandani, Reactive rotational molding, Polymer Engineering and Science 20, 899-919 (1980)

[16] S. D. R. Wilson and J. Williams, The flow of a liquid film on the inside of a rotating cylinder, Phys. Fluids 4, 2184-2190 (1997) 